\title{
Tangential Nevanlinna-Pick Interpolation for Strong Stabilization of MIMO Distributed Parameter Systems
}

\author{
Masashi Wakaiki, Yutaka Yamamoto, and Hitay Özbay
}

\begin{abstract}
We study the problem of finding stable controllers that stabilize a multi-input multi-output distributed parameter system while simultaneously reducing the sensitivity of the system. The plants we consider have finitely many unstable transmission zeros, but they can possess infinitely many unstable poles. Using the tangential Nevanlinna-Pick interpolation with boundary conditions, we obtain both upper and lower bounds of the minimum sensitivity that can be achieved by stable controllers. We also derive a method to find stable controllers for sensitivity reduction. In addition, we apply the proposed method to a repetitive control system.
\end{abstract}

\section{INTRODUCTION}

In this paper, we study sensitivity reduction by stable stabilizing controllers, i.e., strong stabilization with sensitivity reduction, for multi-input multi-output distributed parameter systems. It is desirable to implement stable controllers from the viewpoint of the integrity of the closed-loop systems [5] and the saturation of the control input [27]. Stable controllers are used for control of flexible structures [2], magnetic bearing systems [25], traffic networks [27], and so on.

For finite dimensional systems, many methods have been developed for finding stable $H^{\infty}$ controllers; see, e.g., [11], [16], [22], [32] and their references. For infinite dimensional systems, some works have also been reported recently [12], [13], [20], [28]. Moreover, it was proved in [24] that every stabilizable linear multi-input multi-output plant is strongly stabilizable. However, strong $H^{\infty}$ stabilization for multiinput multi-output distributed parameter systems is still largely open.

In [28], for a class of systems with infinitely many unstable poles, strong stabilization with sensitivity reduction is transformed to the matrix-valued Nevanlinna-Pick interpolation with boundary conditions. This technique leads to a strict assumption that all unstable zeros of the plant must be blocking zeros. In this paper, using the tangential Nevanlinna-Pick interpolation with boundary conditions [1], we obtain both upper and lower bounds on the minimum of the sensitivity that can be achieved by strongly stabilizing controllers. We can handle distributed parameter systems with finitely many unstable transmission zeros and infinitely many unstable poles via the tangential interpolation.

It is well known that the tangential Nevanlinna-Pick interpolation with boundary conditions is solvable if and only

M. Wakaiki and Y. Yamamoto are with the Department of Applied Analysis and Complex Dynamical Systems, Graduate School of Informatics, Kyoto University, Kyoto 606-8501, Japan (e-mail: wakaiki@acs.i.kyoto-u.ac.jp; yy@i.kyoto-u.ac.jp).

H. Özbay is with the Department of Electrical and Electronics Engineering, Bilkent University, Bilkent, Ankara TR-06800, Turkey (e-mail: hitay@bilkent.edu.tr). if the Pick matrix consisting of the interior conditions is positive definite [1]. Techniques to find the solutions are also studied [1], [17]. Thus we can calculate the upper and lower bounds of the minimum sensitivity by iterative calculations of the Pick matrices. Additionally, we design stable controllers attaining a desired sensitivity level.

This paper is organized as follows: Section II gives the statement of the sensitivity reduction problem with stable controllers. In Section III, we transform this problem to a tangential interpolation with an $H^{\infty}$ condition by unimodular matrices in $\mathbf{M}\left(H^{\infty}\right)$. We propose an algorithm for finding stable controllers that achieve low sensitivity in Section IV. We give a numerical example and apply the proposed method to a repetitive control system in Section V. Concluding remarks are drawn in Section VI.

\section{Notation}

Let $\mathbb{C}_{+}$and $\overline{\mathbb{C}}_{+}$denote the open right half-plane $\{s \in$ $\mathbb{C} \mid \operatorname{Re} s>0\}$ and the closed right half-plane $\{s \in$ $\mathbb{C} \mid \operatorname{Re} s \geq 0\}$, respectively.

$H^{\infty}$ denotes the set of functions that are bounded and analytic in $\mathbb{C}_{+}$, and $R H^{\infty}$ denotes the subset of $H^{\infty}$ consisting of rational functions with real coefficients. We denote by $F^{\infty}$ the field of fractions of $H^{\infty}$.

$\mathbf{M}(R)$ is used as a generic symbol to denote the set of matrices with elements in a commutative ring $R$, of whatever size. When it is necessary to show explicitly the size of a matrix, the notation $M \in R^{p \times q}$ is used to indicate that $M$ is a $p \times q$ matrix with entries in $R$.

$M^{\text {adj }}$ and $\operatorname{det} M$ denote the classical adjoint and the determinant of $M \in R^{p \times p}$, respectively.

$M^{*}$ denotes the conjugate transpose of $M \in \mathbf{M}(\mathbb{C})$. The Euclidean norm of $v \in \mathbb{C}^{p}$ is defined by $\|v\|:=\left(v^{*} v\right)^{1 / 2}$, and the Euclidean induced norm of $M \in \mathbb{C}^{p \times q}$ is defined by $\|M\|:=\sup \left\{\|M v\| /\|v\|: v \in \mathbb{C}^{q}\right.$ with $\left.v \neq 0\right\}$, which is equal to the largest singular value of $M$. For $G \in \mathbf{M}\left(H^{\infty}\right)$, the $H^{\infty}$ norm is defined as $\|G\|_{\infty}:=\sup _{s \in \mathbb{C}_{+}}\|G(s)\|$.

\section{PROBLEM STATEMENT}

Consider the linear, continuous-time, time-invariant closed-loop system given in Fig. 1. Let the plant $P$ and the controller $C$ belong to $\mathbf{M}\left(F^{\infty}\right)$. The closed-loop system in Fig. 1 is internally stable if the transfer matrix $H(P, C)$ from $u_{1}, u_{2}$ to $e_{1}, e_{2}$ satisfies

$$
\begin{aligned}
H & (P, C) \\
\quad & =\left[\begin{array}{cc}
(I+P C)^{-1} & -(I+P C)^{-1} P \\
C(I+P C)^{-1} & I-C(I+P C)^{-1} P
\end{array}\right] \in \mathbf{M}\left(H^{\infty}\right) .
\end{aligned}
$$




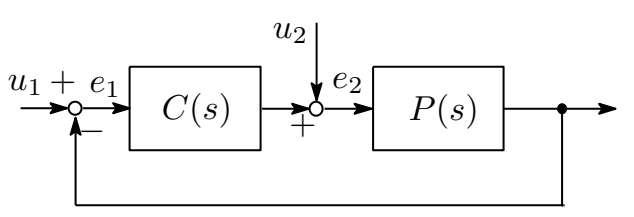

Fig. 1. Closed-loop system.

We say that $C$ stabilizes $P$ and $P$ is stabilizable if the closedloop system is internally stable. Let $\mathscr{C}(P)$ represent the set of all controllers stabilizing $P . P$ is strongly stabilizable if $\mathscr{C}(P)$ contains a stable controller, i.e., $\mathbf{M}\left(H^{\infty}\right) \cap \mathscr{C}(P) \neq \emptyset$.

Our problem in this paper is stated as follows:

Problem 2.1: Given a plant $P \in \mathbf{M}\left(F^{\infty}\right)$, weighting matrices $W_{1}, W_{2} \in \mathbf{M}\left(H^{\infty}\right)$, and $\rho>0$, determine whether there exists a controller $C \in \mathbf{M}\left(H^{\infty}\right) \cap \mathscr{C}(P)$ such that

$$
\left\|W_{1}(I+P C)^{-1} W_{2}\right\|_{\infty}<\rho .
$$

Also, if one exists, find such a controller.

The purpose of the present paper is to give a sufficient condition for the solvability of Problem 2.1 under some assumptions. We also propose a design method for such a controller.

Problem 2.1 is the same as in [28]. The difference is assumptions on the plant. In [28], all the unstable zeros of the plant are blocking zeros. On the other hand, in this paper, we allow that the unstable zeros are transmission zeros.

\section{SENSITIVITY REDUCTION B Y STABLE CONTROLLERS}

In this section, we assume that the plant has only finitely many unstable transmission zeros. Then we show that Problem 2.1 is equivalent to the problem of finding a unimodular matrix $F, F^{-1} \in \mathbf{M}\left(H^{\infty}\right)$ satisfying $\|F\|_{\infty}<\rho$ and finitely many tangential interpolation conditions:

$$
\xi^{*} F\left(s_{i}\right)=\eta_{i}^{*}, \quad i=1, \ldots, n .
$$

This interpolation problem is similar to the tangential Nevanlinna-Pick interpolation problem [1], but the solution needs to be unimodular in $\mathbf{M}\left(H^{\infty}\right)$. In what follows, the notation $\left(s_{i},\left[\xi_{i}, \eta_{i}\right]\right)_{i=1}^{n}$ is used to indicate the tangential interpolation data as in (III.1), i.e., an associating vector pair $\left[\xi_{i}, \eta_{i}\right]$ at $s_{i}$.

On the other hand, in [28], the matrix-valued interpolation conditions $F\left(s_{i}\right)=A_{i}$ are considered. These conditions lead to the strict assumption that the plant has only blocking zeros as its unstable zeros. The advantage of the tangential interpolation is that we can allow unstable transmission zeros. We show that Problem 2.1 can be transformed to the tangential interpolation problem using a similar approach developed in [28], though with some nontrivial modifications.

Let us first study strong stabilization only. The following lemma gives a necessary and sufficient condition for strong stabilization.

Lemma 3.1 ( [28]): Let $P \in \mathbf{M}\left(F^{\infty}\right)$ be stabilizable. Suppose that $P$ has the form $P=D^{-1} N$, where $D$,
$N \in \mathbf{M}\left(H^{\infty}\right)$ are strongly left coprime in the sense of [26], i.e., there exist $X, Y \in \mathbf{M}\left(H^{\infty}\right)$ such that

$$
N X+D Y=I .
$$

Then $P$ is strongly stabilizable if and only if there exists $C \in \mathbf{M}\left(H^{\infty}\right)$ such that

$$
(D+N C)^{-1} \in \mathbf{M}\left(H^{\infty}\right) .
$$

Lemma 3.1 suggests the following problem to find stable stabilizing controllers.

Problem 3.2: Given $D, N \in \mathbf{M}\left(H^{\infty}\right)$, find $C \in \mathbf{M}\left(H^{\infty}\right)$ satisfying (III.3).

Under the following assumption on $D$ and $N$, we can transform Problem 3.2 to a tangential interpolation by a unimodular matrix.

Assumption 3.3: $D, N \in \mathbf{M}\left(H^{\infty}\right)$ are strongly left coprime. All elements of $N, D, X$, and $Y$ in (III.2) are meromorphic in $\mathbb{C}$.

In addition, $N$ is square and $\operatorname{det} N$ has the form $\operatorname{det} N=$ $\phi N_{o}$, where $\phi \in R H^{\infty}$ and $N_{o}, 1 / N_{o} \in H^{\infty}$. The rational function $\phi$ satisfies $\phi(\infty) \neq 0$ and possesses simple zeros $z_{1}, \ldots, z_{n}$ in $\overline{\mathbb{C}}_{+}$. For $i=1, \ldots, n$, nonzero $v_{i} \in \mathbb{C}^{p}$ satisfying

$$
v_{i}^{*} N\left(z_{i}\right)=0
$$

is unique to within multiplication by a constant complex number.

Note that $\operatorname{det} N$ in Assumption 3.3 has no pure delay term $e^{-h s}$ for any $h>0$.

Under Assumption 3.3, we see that Problem 3.2 is equivalent to the following problem:

Problem 3.4: Suppose that $s_{1}, \ldots, s_{n} \in \overline{\mathbb{C}}_{+}$are distinct and that $\xi_{1}, \ldots, \xi_{n}, \eta_{1}, \ldots, \eta_{n} \in \mathbb{C}^{p}$. Find a unimodular matrix $U, U^{-1} \in\left(H^{\infty}\right)^{p \times p}$ such that all elements of $U$ are meromorphic in $\mathbb{C}$ and

$$
\xi_{i}^{*} U\left(s_{i}\right)=\eta_{i}^{*}, \quad i=1, \ldots, n .
$$

The following result reduces strong stabilization to a tangential interpolation by a unimodular matrix.

Theorem 3.5: Consider Problem 3.2 under Assumption 3.3. We restrict the solutions to matrices whose entries are meromorphic in $\mathbb{C}$. Then Problem 3.2 is equivalent to Problem 3.4 with $\left(z_{i},\left[v_{i}, D\left(z_{i}\right)^{*} v_{i}\right]\right)_{i=1}^{n}$.

Furthermore, a solution $C$ of Problem 3.2 and a solution $U$ of Problem 3.4 satisfy the following equation:

$$
C=N^{-1}(U-D), \quad U=D+N C
$$

Proof: For the proof, see the appendix.

Prasanth [23] presents a method to find a unimodular matrix satisfying tangential interpolation conditions. In [23], a result similar to Theorem 3.5 is developed for finite dimensional systems. The augment of [23] is based on a state-space realization of the plant, but we prove Theorem 3.5 in a transfer function approach.

Before we proceed to strong stabilization with sensitivity reduction, we need to recall the definitions of co-inner matrix and co-outer matrix functions. $F \in \mathbf{M}\left(H^{\infty}\right)$ is co-inner if $F(\bar{s})^{*}$ is inner. $G \in \mathbf{M}\left(H^{\infty}\right)$ is co-outer if $G(\bar{s})^{*}$ is outer. 
Every function in $\mathbf{M}\left(H^{\infty}\right)$ admits a unique co-inner-outer factorization.

Theorem 3.6 ( [6]): Let $K$ be in $\left(H^{\infty}\right)^{p \times q}$. $K$ admits a co-inner-outer factorization of the form $K=G F$, where $G \in\left(H^{\infty}\right)^{p \times r}$ is co-outer and $F \in\left(H^{\infty}\right)^{r \times q}$ is co-inner for some $r$. In addition, $F$ and $G$ are unique to within multiplication by a constant unitary matrix.

Let us next consider Problem 2.1. We place this additional assumption on $W_{1}, W_{2}$, and $D$ :

Assumption 3.7: All elements of $W_{1}$ and $W_{2}$ are meromorphic functions in $\mathbb{C}$. Both $W_{1}$ and $W_{1}^{-1}$ are in $\mathbf{M}\left(H^{\infty}\right)$. When we factorize $D W_{2}$ in the form $D W_{2}=\left(D W_{2}\right)_{c o}$. $\left(D W_{2}\right)_{c i}$, where $\left(D W_{2}\right)_{c o}$ is co-outer and $\left(D W_{2}\right)_{c i}$ is coinner, $\left(D W_{2}\right)_{\text {co }}$ as well as $\left(D W_{2}\right)_{\text {co }}^{-1}$ are in $\mathbf{M}\left(H^{\infty}\right)$.

We can obtain a solution for Problem 2.1 under Assumption 3.3 and 3.7 , using a solution of the following problem. The only difference from Problem 3.4 is to have a simple $H^{\infty}$ norm condition.

Problem 3.8: Suppose that $s_{1}, \ldots, s_{n} \in \overline{\mathbb{C}}_{+}$are distinct, and that $\xi_{1}, \ldots, \xi_{n}, \eta_{1}, \ldots, \eta_{n}$ are in $\mathbb{C}^{p}$. Suppose also that $\rho>0$. Find a unimodular matrix $F, F^{-1} \in\left(H^{\infty}\right)^{p \times p}$ such that all elements of $F$ are meromorphic in $\mathbb{C},\|F\|_{\infty}<\rho$, and

$$
\xi_{i}^{*} F\left(s_{i}\right)=\eta_{i}^{*}, \quad i=1, \ldots, n .
$$

Theorem 3.9: Consider Problem 2.1. Suppose that there exist $D, N \in \mathbf{M}\left(H^{\infty}\right)$ such that $P=D^{-1} N$. Let Assumption 3.3 and 3.7 hold. Define

$$
\begin{aligned}
\xi_{i} & :=\left(D\left(z_{i}\right) W_{1}^{-1}\left(z_{i}\right)\right)^{*} v_{i}, \\
\eta_{i} & :=\left(\left(D W_{2}\right)_{c o}\left(z_{i}\right)\right)^{*} v_{i}, \quad i=1, \ldots, n .
\end{aligned}
$$

If there exists a solution $F$ of Problem 3.8 with $\left(z_{i},\left[\xi_{i}, \eta_{i}\right]\right)_{i=1}^{n}$ and $\rho$, then

$$
C:=N^{-1}\left(D W_{2}\right)_{c o} F^{-1} W_{1}-P^{-1}
$$

gives a solution of Problem 2.1. Conversely, if there exists a meromorphic solution $C$ of Problem 2.1, then

$$
F:=W_{1}(D+N C)^{-1}\left(D W_{2}\right)_{c o}
$$

is a solution of Problem 3.8 with $\left(z_{i},\left[\xi_{i}, \eta_{i}\right]\right)_{i=1}^{n}$ and $\rho$.

Proof: For the proof, see the appendix.

Theorem 3.9 suggests that the problem of strong stabilization with sensitivity reduction is equivalent to Problem 3.8. Problem 3.8 is also difficult to solve, but it is easy to obtain both a sufficient condition and a necessary condition for Problem 3.8. In the next section, we remove the condition $F^{-1} \in\left(H^{\infty}\right)^{p \times p}$ and then obtain a sufficiency of Problem 2.1 by the tangential Nevanlinna-Pick interpolation. Before proceeding to the next section, we formulate the necessary condition, which is also derived by the tangential Nevanlinna-Pick interpolation.

Corollary 3.10: Consider Problem 2.1 under the same hypotheses of Theorem 3.9. Suppose that Problem 2.1 whose solutions are restricted to meromorphic matrices is solvable. Then there exists $F \in \mathbf{M}\left(H^{\infty}\right)$ such that $\|F\|_{\infty}<1$ and

$$
\rho \xi_{i}^{*} F\left(z_{i}\right)=\eta_{i}^{*}, \quad i=1, \ldots, n .
$$

Proof: It is obvious from Theorem 3.9.

Remark 3.11: In this section, we assume that all $H^{\infty}$ functions are meromorphic in $\mathbb{C}$ because $H^{\infty}$ functions do not have a fixed value on the imaginary axis. If the unstable zeros of $\operatorname{det} N$ are not on the imaginary axis in Assumption 3.3 , then we do not need the assumption that all elements of transfer matrices are meromorphic.

\section{DESIGN OF STABLE CONTROLLERS}

In this section, we derive a design method of strongly stabilizing controllers for sensitivity reduction, extending the technique of [16], [28] to the tangential interpolation case.

The design method is based on the following lemma.

Lemma 4.1 ( [16], [28]): Suppose that $G \in\left(H^{\infty}\right)^{p \times p}$ and that $\|G\|_{\infty}<1$. Then, for every complex number $\lambda \neq 0$,

$$
F:=\frac{\lambda}{2}(G+I)
$$

satisfies $F, F^{-1} \in \mathbf{M}\left(H^{\infty}\right)$ and $\|F\|_{\infty}<|\lambda|$.

We can remove the condition $F^{-1} \in\left(H^{\infty}\right)^{p \times p}$ in Problem 3.8 by Lemma 4.1 . Thus we obtain the following sufficient condition for Problem 3.8:

Theorem 4.2: Consider Problem 3.8. Let $\lambda \in \mathbb{C}$ satisfy $|\lambda|=\rho$. Define

$$
\zeta_{i}:=\frac{2}{\bar{\lambda}} \eta_{i}-\xi_{i}, \quad i=1, \ldots, n .
$$

If $G \in \mathbf{M}\left(H^{\infty}\right)$ satisfies $\|G\|_{\infty}<1$ and

$$
\xi_{i}^{*} G\left(z_{i}\right)=\zeta_{i}^{*}, \quad i=1, \ldots, n,
$$

then $F$ defined by (IV.1) is a solution of Problem 3.8.

Proof: It follows from Lemma 4.1 that $F$ and $F^{-1}$ belong to $\left(H^{\infty}\right)^{p \times p}$ and that $\|F\|_{\infty}<\rho$. By (IV.1) and (IV.2), $F$ satisfies the interpolation conditions (III.6).

The problem of finding $G$ in Theorem 4.2 and that of finding $F$ in Corollary 3.10 are the following tangential Nevanlinna-Pick interpolation with boundary conditions:

Problem 4.3 ( [1]): Given distinct $\alpha_{1}, \ldots \alpha_{n} \in \mathbb{C}_{+}$, $j \omega_{1}, \ldots, j \omega_{m} \in j \mathbb{R}$, and vector pairs

$$
\left\{\left[\xi_{i}, \eta_{i}\right]\right\}_{i=1}^{n}, \quad\left\{\left[x_{k}, y_{k}\right]\right\}_{k=1}^{m} \subset \mathbb{C}^{p} \times \mathbb{C}^{q}
$$

satisfying

$$
\begin{aligned}
& \left\|\xi_{i}\right\|-\left\|\eta_{i}\right\|>0, \quad i=1, \ldots, n, \\
& \left\|x_{k}\right\|-\left\|y_{k}\right\|>0, \quad k=1, \ldots, m,
\end{aligned}
$$

find $\Phi \in\left(H^{\infty}\right)^{p \times q}$ satisfying $\|\Phi\|_{\infty}<1$ and

$$
\begin{aligned}
& \xi_{i}^{*} \Phi\left(\alpha_{i}\right)=\eta_{i}^{*}, \quad i=1, \ldots, n, \\
& x_{k}^{*} \Phi\left(j \omega_{k}\right)=y_{k}^{*}, \quad k=1, \ldots, m .
\end{aligned}
$$

It is well known that Problem 4.3 is solvable if and only if the Pick matrix consisting of the interior conditions is positive definite.

Theorem 4.4 ( [1]): Consider Problem 4.3. Define the Pick matrix

$$
Q:=\left[\begin{array}{ccc}
Q_{11} & \cdots & Q_{1 n} \\
\vdots & & \vdots \\
Q_{n 1} & \cdots & Q_{n n}
\end{array}\right]
$$


where

$$
Q_{k l}:=\frac{\xi_{k}^{*} \xi_{l}-\eta_{k}^{*} \eta_{l}}{\alpha_{k}+\bar{\alpha}_{l}}, \quad k, l=1, \ldots, n .
$$

Then Problem 4.3 is solvable if and only if $Q$ is positive definite.

We have shown in Theorem 4.2 and Corollary 3.10 that both a sufficient condition and a necessary condition for Problem 2.1 can be reduced to the solvability of (different) Problem 4.3. Hence by checking whether the associate Pick matrices are positive definite, we can calculate a lower and upper bound of the minimum sensitivity that can be achieved by stable controllers. In addition, techniques to find the solutions are well studied in [1], [17], so we also construct a stable controller attaining low sensitivity by the following algorithm:

\section{A solution to Problem 2.1}

Step 1: Let $\lambda \in \mathbb{C}$ satisfy $|\lambda|=\rho$. Let the interpolation conditions of $G$ be defined as follows:

$$
\xi_{i}^{*} G\left(z_{i}\right)=\zeta_{i}^{*}, \quad i=1, \ldots, n,
$$

where

$$
\begin{aligned}
\xi_{i} & :=\left(D\left(z_{i}\right) W_{1}^{-1}\left(z_{i}\right)\right)^{*} v_{i}, \\
\zeta_{i} & :=\frac{2}{\bar{\lambda}}\left(\left(D W_{2}\right)_{c o}\left(z_{i}\right)\right)^{*} v_{i}-\xi_{i} .
\end{aligned}
$$

Step 2: Solve the tangential Nevanlinna-Pick interpolation problem with boundary conditions of $G$.

Step 3: Calculate a solution of Problem 3.8 by (IV.1).

Step 4: Compute a stable controller attaining low sensitivity by (III.7).

Remark 4.5: As in almost all works on stable $H^{\infty}$ control, our design technique is based on the sufficient condition. We use the small gain theorem and the triangle inequality in Lemma 4.1.

We should confirm that the set of the controllers obtained by the proposed method become smaller as $\rho$ in (II.2) decreases. The following proposition ensures the property.

Proposition 4.6: Let $\left\{\lambda_{k}\right\}_{k \geq 1} \subset \mathbb{C}$ satisfy $\lambda_{1} \neq 0$. Assume that for every $k \geq 1$, there exists $L_{k} \in(0,1]$ such that $\lambda_{k+1}=L_{k} \lambda_{k}$. Suppose that $z_{1}, \ldots, z_{n} \in \overline{\mathbb{C}}_{+}$ are distinct and that $\xi_{1}, \ldots, \xi_{n}$ and $\eta_{1}, \ldots, \eta_{n}$ are in $\mathbb{C}^{p}$. Suppose also that $\mathscr{N}(\lambda)$ is the set whose elements are the solutions of Problem 4.3 with the following interpolation conditions:

$$
\xi_{i}^{*} G\left(z_{i}\right)=\frac{2}{\lambda} \eta_{i}^{*}-\xi_{i}^{*}, \quad i=1, \ldots, n .
$$

Define

$$
\mathscr{M}\left(\lambda_{k}\right):=\left\{\frac{\lambda_{k}}{2}\left(G_{k}+I\right): G_{k} \in \mathscr{N}\left(\lambda_{k}\right)\right\} .
$$

Then we have

$$
\mathscr{M}\left(\lambda_{k+1}\right) \subset \mathscr{M}\left(\lambda_{k}\right) .
$$

Proof: For the proof, see the appendix.

In general, the proposed method gives an infinite dimensional controller. To obtain an implementable controller, we must approximate the controller derived by the proposed method.

The following results tells us that a rational stable controller also stabilizes the plant and achieves low sensitivity of the closed-loop system if the infinite dimensional controller is enough approximated by the rational controller in the sense of $H^{\infty}$ norm. These results are the extension of the scalar case in [9].

Proposition 4.7: Let $P$ be in $\mathbf{M}\left(F^{\infty}\right)$. Suppose that there exist $D, N \in \mathbf{M}\left(H^{\infty}\right)$ such that $P=D^{-1} N$ and $D$, $N$ are strongly left coprime. For $C \in \mathbf{M}\left(H^{\infty}\right) \cap \mathscr{C}(P)$, if $C_{a} \in \mathbf{M}(R H)^{\infty}$ satisfies

$$
\left\|C-C_{a}\right\|_{\infty}<\epsilon:=\frac{1}{\|N\|_{\infty} \cdot\left\|(D+N C)^{-1}\right\|_{\infty}},
$$

then $C_{a}$ also stabilizes $P$.

Proof: For the proof, see the appendix.

Proposition 4.8: Consider Problem 2.1. Suppose that both $W_{1}$ and $W_{1}^{-1}$ are in $\mathbf{M}\left(H^{\infty}\right)$. For $C \in \mathbf{M}\left(H^{\infty}\right) \cap$ $\mathscr{C}(P)$ and $C_{a} \in \mathbf{M}\left(R H^{\infty}\right) \cap \mathscr{C}(P)$, we define

$$
\begin{aligned}
\delta & :=\left\|W_{1}(I+P C)^{-1} P\right\|_{\infty} \cdot\left\|W_{1}^{-1}\right\|_{\infty}, \\
\epsilon & :=\left\|C-C_{a}\right\|_{\infty}, \\
S & :=(I+P C)^{-1}, \quad S_{a}:=\left(I+P C_{a}\right)^{-1} .
\end{aligned}
$$

If $\delta \epsilon<1$, then

$$
\left\|W_{1} S_{a} W_{2}\right\|_{\infty} \leq \frac{\left\|W_{1} S W_{2}\right\|_{\infty}}{1-\delta \epsilon} .
$$

Proof: For the proof, see the appendix.

Remark 4.9: In Proposition 4.8, $\left\|W_{1}^{-1}\right\|_{\infty}$ in (IV.5) may make the estimation (IV.6) conservative. Since $W_{1}$ is not generally commutative, it is difficult to get rid of $W_{1}$ and $W_{1}^{-1}$ in (IV.5). However, if $W_{1}$ is a scalar matrix, i.e., a diagonal matrix whose diagonal elements contain the same scalar function, then we can change (IV.5) to

$$
\delta:=\left\|(I+P C)^{-1} P\right\|_{\infty} .
$$

See in [21] and the references therein for the details of the approximation techniques.

\section{NUMERICAL EXAMPLES}

In this section, we present a numerical example to show the efficiency of the results. We also apply the proposed method to a repetitive control system [14], [31].

Example 5.1: We consider sensitivity reduction by strongly stabilizing controllers for the following distributed parameter system and weighting functions:

$$
\begin{gathered}
P(s)=\left[\begin{array}{cc}
\frac{\left(s-z_{1}\right)\left(s-z_{2}\right)}{(s+1)^{2}\left(3+4 e^{-s}\right)} & e^{-2 s} \\
0 & \frac{(s+1)^{2}}{(s-1 / 2)\left(s-e^{-s}+2\right)}
\end{array}\right], \\
W_{1}(s)=\frac{s+1}{10 s+1} I, \quad W_{2}(s)=I,
\end{gathered}
$$

where $z_{1}, z_{2} \in \overline{\mathbb{C}}_{+}$are distinct.

First we find $D, N \in \mathbf{M}\left(H^{\infty}\right)$ satisfying the conditions of Assumption 3.3. Using the factorization method of [13] 


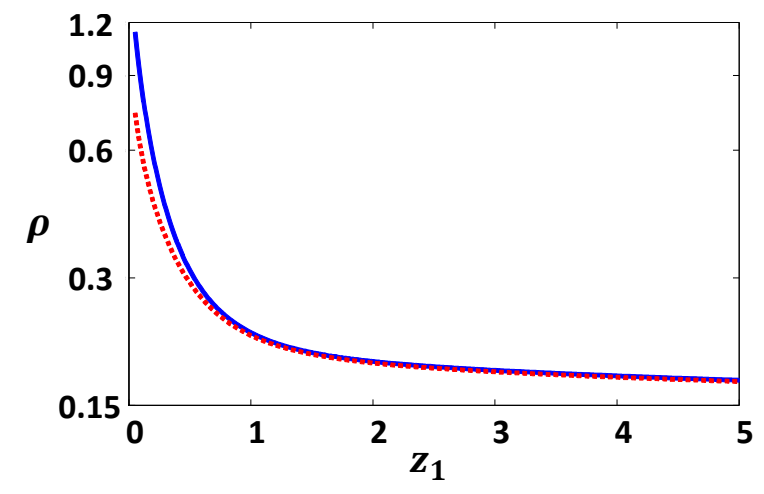

Fig. 2. $\rho$ versus $z_{1}$.

to each elements of $P, P$ can be factorized as $P=D^{-1} N$, where

$$
\begin{gathered}
D(s):=\left[\begin{array}{cc}
\frac{3+4 e^{-s}}{3 e^{-s}+4} & 0 \\
0 & \frac{s-1 / 2}{s+1 / 2}
\end{array}\right], \\
N(s):=\left[\begin{array}{cc}
\frac{\left(s-z_{1}\right)\left(s-z_{2}\right)}{(s+1)^{2}\left(3 e^{-s}+4\right)} & \frac{3+4 e^{-s}}{3 e^{-s}+4} e^{-2 s} \\
0 & \frac{(s+1)^{2}}{(s+1 / 2)\left(s-e^{-s}+2\right)}
\end{array}\right] .
\end{gathered}
$$

The zeros of $\operatorname{det} N$ in $\overline{\mathbb{C}}_{+}$are $z_{1}$ and $z_{2}$. Furthermore, $v_{i}:=\left[\begin{array}{ll}-\frac{3 e^{-z_{i}}+4}{\left(3+4 e^{-z_{i}}\right) e^{-2 z_{i}}} & \frac{\left(z_{i}+1 / 2\right)\left(z_{i}-e^{-z_{i}}+2\right)}{\left(z_{i}+1\right)^{2}}\end{array}\right]^{*}, \quad i=1,2$, satisfies $v_{i}^{*} N\left(z_{i}\right)=0$ and $v_{i}$ is unique to within multiplication by a constant complex number.

It can be proved in the same way as Theorem 3.5 that $D$ and $N$ are strongly left coprime if and only if there exists $Y \in \mathbf{M}\left(H^{\infty}\right)$ such that $Y$ satisfies the following interpolation conditions:

$$
v_{i}^{*} D\left(z_{i}\right) Y\left(z_{i}\right)=v_{i}^{*}, \quad i=1,2 .
$$

In addition, we can check the existence of $Y$ satisfying these interpolation conditions by Theorem 4.4.

We take $0<z_{1} \leq 5$ and $z_{2}=8$. Fig. 2 shows the relationship between the sensitivity $\rho$ in (II.2) and the unstable transmission zero $z_{1}$. In Fig. 2, the solid line indicates the minimum of $\rho$ obtained by the proposed method, and the dashed line shows a lower bound of $\rho$ achieved by stable controllers. The lower bound is derived in Corollary 3.10. From Fig. 2, we see that an unstable pole-zero cancellation at $s=1 / 2$ in $\operatorname{det} P$ does not affect strong stabilization with sensitivity reduction in this example. This is because $z_{1}$ is not a blocking zero.

Example 5.2: (Application to repetitive control systems) Consider the repetitive control system given in Fig. 3. Repetitive control intends to track or reject arbitrary periodic signals of a fixed period. It is well known that repetitive control is effective for control of industrial robotic manipulators [3] and disc drives [18]. In addition, repetitive control systems have been recently applied to DC-AC converters in microgrids [29], shunt active power filters [10], wind turbines [15], and so on.

The well-known internal model principle [8] is extended to the class of psedorational impulse response matrices [31].

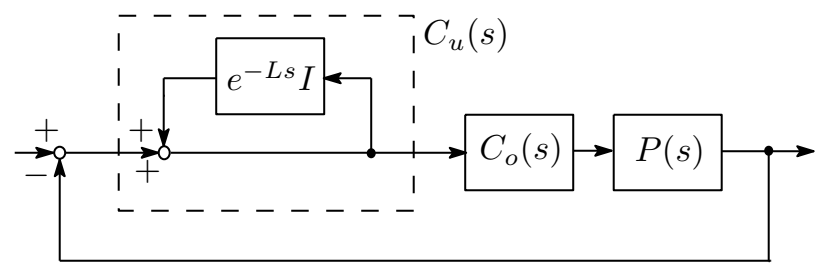

Fig. 3. Repetitive control system.

It is proved in [31] that exponential decay of the error signal for any reference signal with a fixed period $L$ is equivalent to the existence of the internal model $1 /\left(1-e^{-L s}\right)$ under the condition of exponential stability of the closed-loop system.

By this principle, the controllers we study can be separated into two parts $C=C_{u} C_{o}$, where $C_{u}$ is the part of the internal model $1 /\left(1-e^{-L s}\right) \cdot I$ and $C_{o}$ is the stable part to be designed. For the design of $C_{o}$, we can consider the product $C_{u} P=: P_{o}$ to be the new plant to be controlled.

As we will discuss in Theorem 5.3 and a paragraph after it, $P$ should not have zeros on the imaginary axis for the stabilizability of $P_{o}$. However, $P$ is allowed to have transmission zeros in $\mathbb{C}_{+}$under certain assumptions. Note that this example is different from that in [28], where the unstable zeros of $P$ need to be blocking zeros.

To guarantee exponential stability, it is necessary that $H(P, C)$ in (II.1) has no poles in the region $\mathbb{C}_{-\varepsilon}:=\{s \in$ $\mathbb{C} \mid \operatorname{Re} s \geq-\varepsilon\}$, where $\varepsilon>0$ is fixed [30]. Thus our objective is finding $\tilde{C} \in \mathbf{M}\left(H^{\infty}\right)$ that stabilizes

$$
\tilde{P}(s):=P_{o}(s-\varepsilon)=C_{u}(s-\varepsilon) P(s-\varepsilon),
$$

which has an infinitely many unstable poles in $\mathbb{C}_{+}$, while simultaneously reducing the sensitivity of the closed-loop system. Once we find such a $\tilde{C}$, we determine the stable part $C_{o}(s):=\tilde{C}(s+\varepsilon)$. Since $\tilde{C}$ is in $\mathbf{M}\left(H^{\infty}\right), C_{o}$ does not have poles in $\mathbb{C}_{-\varepsilon}$.

Let the plant $P$ be a finite dimensional system. In general, it is difficult to obtain a strongly left coprime factorization of multi-input multi-output distributed parameter systems. However, the only distributed parameter part $C_{u}$ of $\tilde{P}$ is scalar. Hence we can construct a strongly left coprime factorization of $\tilde{P}$ by a left coprime factorization of $P$.

Theorem 5.3: Suppose that $D, N \in\left(R H^{\infty}\right)^{p \times p}$ satisfy the conditions of Assumption 3.3. Let $f \in H^{\infty}$ satisfy $f\left(z_{i}\right) \neq 0$ for every $i$. Then $f D$ and $N$ are strongly left coprime.

Proof: For the proof, see the appendix.

Theorem 5.3 suggests that under some assumptions on unstable transmission zeros of $P, \tilde{P}$ defined by (V.1) has a strongly left coprime factorization

$$
\tilde{P}(s)=\left(\frac{1-e^{L \varepsilon} e^{-L s}}{e^{-L s}-e^{L \varepsilon}} D\right)^{-1} \cdot\left(\frac{1}{e^{-L s}-e^{L \varepsilon}} N\right),
$$

where $D, N \in\left(R H^{\infty}\right)^{p \times p}$ are left coprime and satisfy $P(s-\varepsilon)=D^{-1}(s) N(s)$. Roughly speaking, this means that we can obtain a strongly left coprime factorization of $\tilde{P}$ by a left coprime factorization of $P$ if there are no unstable hidden modes in the product $\tilde{P}=C_{u} P$. 
As a numerical example, we take $\varepsilon:=0.1, L:=1, W_{2}=$ $I$, and

$$
P(s):=\left[\begin{array}{cc}
\frac{s-5}{s-1 / 10} & \frac{1}{s-1 / 10} \\
\frac{2}{s+2} & \frac{s-1}{s+1}
\end{array}\right], \quad W_{1}(s):=\frac{s+1}{10 s+1} I .
$$

We study Problem 2.1 for $\tilde{P}$ in (V.1), $W_{1}$, and $W_{2}$.

The minimum of $\rho$ derived by the proposed method is 0.2632. A solution $\tilde{C} \in \mathbf{M}\left(H^{\infty}\right)$ of Problem 2.1 with $\rho=$ 0.2632 is given by $\tilde{C}=N^{-1} D_{c o} F^{-1} W_{1}-\tilde{P}$, where $D_{c o}$ is a co-outer matrix of $D$ and

$$
F(s) \approx\left[\begin{array}{ll}
\frac{0.1503(s+5.163)}{s+5.467} & \frac{0.0508(s+1.906)}{s+5.467} \\
\frac{0.02783(s+5.949)}{s+5.467} & \frac{0.2484(s+5.681)}{s+5.467}
\end{array}\right] .
$$

On the other hand, we obtain a lower bound of the minimum sensitivity that can be achieved by a stable controller, 0.2629 by Corollary 3.10 .

\section{CONCLUDING REMARKS}

We have studied strong stabilization with sensitivity reduction for a linear time-invariant multi-input multi-output distributed parameter system. The system we consider has only finitely many simple unstable transmission zeros but it is allowed to have infinitely many unstable poles. This problem has not yet been completely solved. However, by the tangential Nevanlinna-Pick interpolation and the associated Pick matrix, we have obtained both upper and lower bounds of the minimum sensitivity that can be attained by stable controllers. We have also proposed a design method of stable controllers for sensitivity reduction. In addition, we have presented a numerical example to illustrate the results and have discussed a repetitive control system as an application of the proposed method.

\section{APPENDIX}

Proof of Theorem 3.5: Let $C \in \mathbf{M}\left(H^{\infty}\right)$ be a meromorphic solution of Problem 3.2. Define $U:=D+N C$. Then $U$ satisfies $U, U^{-1} \in \mathbf{M}\left(H^{\infty}\right)$ by Lemma 3.1 and

$$
\begin{aligned}
v_{i}^{*} U\left(z_{i}\right) & =v_{i}^{*} D\left(z_{i}\right)+v_{i}^{*} N\left(z_{i}\right) C\left(z_{i}\right) \\
& =v_{i}^{*} D\left(z_{i}\right)=\left(D\left(z_{i}\right)^{*} v_{i}\right)^{*} .
\end{aligned}
$$

Thus $U$ must be a solution to Problem 3.4. with the data $\left(z_{i},\left[v_{i}, D\left(z_{i}\right)^{*} v_{i}\right]\right)_{i=1}^{n}$.

Conversely, suppose that there exists $U, U^{-1} \in \mathbf{M}\left(H^{\infty}\right)$ solving Problem 3.4 with $\left(z_{i},\left[v_{i}, D\left(z_{i}\right)^{*} v_{i}\right]\right)_{i=1}^{n}$. Define $C:=N^{-1}(U-D)$. Then $C$ satisfies $(D+N C)^{-1}=U^{-1} \in$ $\mathbf{M}\left(H^{\infty}\right)$,

$$
N C=U-D \in \mathbf{M}\left(H^{\infty}\right),
$$

and

$$
v_{i}^{*}(N C)\left(z_{i}\right)=v_{i}^{*}\left(U\left(z_{i}\right)-D\left(z_{i}\right)\right)=0 .
$$

We prove $C \in \mathbf{M}\left(H^{\infty}\right)$ by (VI.1) and (VI.2) as follows. Define $\Upsilon:=N C$. We have $\Upsilon \in \mathbf{M}\left(H^{\infty}\right)$ by (VI.1) and $v_{i}^{*} \Upsilon\left(z_{i}\right)=0$ by (VI.2). By the definition of $\Upsilon$ and

$$
N^{\operatorname{adj}} \cdot N=\operatorname{det} N \cdot I
$$

we obtain

$$
\phi C=1 / N_{o} \cdot N^{\mathrm{adj}} \cdot \Upsilon \in \mathbf{M}\left(H^{\infty}\right) .
$$

Furthermore, we can prove

$$
\phi\left(z_{i}\right) C\left(z_{i}\right)=1 / N_{o}\left(z_{i}\right) \cdot N^{\operatorname{adj}}\left(z_{i}\right) \Upsilon\left(z_{i}\right)=0, \quad i=1, \ldots, n
$$

because the $l$-th row of $N^{\text {adj }}\left(z_{i}\right), \quad N_{l}^{\text {adj }}\left(z_{i}\right)$, satisfies $N_{l}^{\operatorname{adj}}\left(z_{i}\right)=k_{l} v_{i}^{*}$ for some $k_{l} \in \mathbb{C}$. In fact, by (VI.3),

$$
N^{\operatorname{adj}}\left(z_{i}\right) N\left(z_{i}\right)=\phi\left(z_{i}\right) I=0,
$$

which leads to $N_{l}^{\text {adj }}\left(z_{i}\right) N\left(z_{i}\right)=0$ for $l=1, \ldots, p$. By Assumption 3.3, $v_{i}$ satisfying (III.4) is unique to within multiplication by a constant, so there exists $k_{l} \in \mathbb{C}$ such that $N_{l}^{\operatorname{adj}}\left(z_{i}\right)=k_{l} v_{i}^{*}$.

Thus it suffices to prove that these three conditions:

- $\phi$ satisfies $\phi(\infty) \neq 0$ and the unstable zeros of $\phi$ are $z_{1}, \ldots, z_{n} \in \overline{\mathbb{C}}_{+}$, which are simple,

- $\phi C \in \mathbf{M}\left(H^{\infty}\right)$ and all elements of $\phi C$ are meromorphic in $\mathbb{C}$,

- $(\phi C)\left(z_{i}\right)=0, \quad i=1, \ldots, n$,

lead to $C \in \mathbf{M}\left(H^{\infty}\right)$. Since $\phi C$ is in $\mathbf{M}\left(H^{\infty}\right)$, if $C$ is not in $\mathbf{M}\left(H^{\infty}\right)$, then $C$ has some poles in $\overline{\mathbb{C}}_{+}$, which are canceled by the zeros of $\phi$. Let $z_{i}$ be one of the poles. Since $z_{i}$ is a simple zero, we have $(\phi C)\left(z_{i}\right) \neq 0$. This contradicts $(\phi C)\left(z_{i}\right)=0$. Thus $C$ is in $\mathbf{M}\left(H^{\infty}\right)$. This completes the proof.

Proof of Theorem 3.9: (Outline only) By Theorem 3.5, we can prove that $C$ is in $\mathbf{M}\left(H^{\infty}\right) \cap \mathscr{C}(P)$ if and only if both $F$ and $F^{-1}$ are in $\mathbf{M}\left(H^{\infty}\right)$ and $F$ satisfies the tangential interpolation conditions. After simple calculations, we also see $\left\|W_{1}(1+P C)^{-1} W_{2}\right\|_{\infty}=\|F\|_{\infty}$.

Proof of Proposition 4.6: Assume that $F \in \mathscr{M}\left(\lambda_{k+1}\right)$. There exists $G_{k+1} \in \mathscr{N}\left(\lambda_{k+1}\right)$ such that

$$
F=\frac{\lambda_{k+1}}{2}\left(G_{k+1}+I\right) \text {. }
$$

Define $G_{k}$ as

$$
G_{k}:=\frac{\lambda_{k+1}}{\lambda_{k}}\left(G_{k+1}+I\right)-I .
$$

Then $G_{k}$ is in $\mathscr{N}\left(\lambda_{k}\right)$. In fact, by (IV.3),

$$
\begin{aligned}
\xi_{i}^{*} G_{k}\left(z_{i}\right) & =\xi_{i}^{*}\left(\frac{\lambda_{k+1}}{\lambda_{k}}\left(G_{k+1}\left(z_{i}\right)+I\right)-I\right) \\
& =\frac{\lambda_{k+1}}{\lambda_{k}}\left(\frac{2}{\lambda_{k+1}} \eta_{i}^{*}-\xi_{i}^{*}\right)+\frac{\lambda_{k+1}-\lambda_{k}}{\lambda_{k}} \xi_{i}^{*} \\
& =\frac{2}{\lambda_{k}} \eta_{i}^{*}-\xi_{i}^{*} .
\end{aligned}
$$

Moreover, since $\left\|G_{k+1}\right\|_{\infty}<1$,

$$
\begin{aligned}
\left\|G_{k}\right\|_{\infty} & =\left\|\frac{\lambda_{k+1}}{\lambda_{k}}\left(G_{k+1}+I\right)-I\right\|_{\infty} \\
& \leq \frac{\left|\lambda_{k+1}\right|}{\left|\lambda_{k}\right|} \cdot\left\|G_{k+1}\right\|_{\infty}+\frac{\left|\lambda_{k+1}-\lambda_{k}\right|}{\left|\lambda_{k}\right|} \\
& <\frac{L_{k}\left|\lambda_{k}\right|+\left(1-L_{k}\right)\left|\lambda_{k}\right|}{\left|\lambda_{k}\right|}=1 .
\end{aligned}
$$


Hence $G_{k} \in \mathscr{N}\left(\lambda_{k}\right)$. By (VI.5) and (VI.6), we also have

$$
F=\frac{\lambda_{k}}{2}\left(G_{k}+I\right) .
$$

Hence $F \in \mathscr{M}\left(\lambda_{k}\right)$. Thus (IV.4) is obtained.

Proof of Proposition 4.7: By Lemma 3.1, it suffices to prove that $U_{a}:=D+N C_{a}$ satisfies $U_{a}^{-1} \in \mathbf{M}\left(H^{\infty}\right)$.

Define $U:=D+N C$, which satisfies $U^{-1} \in \mathbf{M}\left(H^{\infty}\right)$ by Lemma 3.1. Since

$$
\begin{aligned}
\left\|U-U_{a}\right\|_{\infty} & \leq\|N\|_{\infty} \cdot\left\|C-C_{a}\right\|_{\infty} \\
& <\|N\|_{\infty} \cdot \epsilon=1 /\left\|U^{-1}\right\|_{\infty}
\end{aligned}
$$

we have $\left\|I-U^{-1} U_{a}\right\|_{\infty}<1$. This means that both

$$
V:=I-\left(I-U^{-1} U_{a}\right)=U^{-1} U_{a}
$$

and $V^{-1}$ are in $\mathbf{M}\left(H^{\infty}\right)$ by Lemma 4.1. Thus $U_{a}^{-1}=$ $V^{-1} U \in \mathbf{M}\left(H^{\infty}\right)$ is obtained.

\section{Proof of Proposition 4.8: Since}

$$
\begin{aligned}
& W_{1} S W_{2}-W_{1} S_{a} W_{2} \\
& \quad=W_{1}\left((I+P C)^{-1}-\left(I+P C_{a}\right)^{-1}\right) W_{2} \\
& \quad=W_{1}(I+P C)^{-1} P\left(C_{a}-C\right) W_{1}^{-1}\left(W_{1} S_{a} W_{2}\right),
\end{aligned}
$$

we obtain

$$
\begin{aligned}
\left\|W_{1} S_{a} W_{2}\right\|_{\infty}-\left\|W_{1} S W_{2}\right\|_{\infty} & \leq\left\|W_{1} S W_{2}-W_{1} S_{a} W_{2}\right\|_{\infty} \\
& \leq \delta \epsilon\left\|W_{1} S_{a} W_{2}\right\|_{\infty} .
\end{aligned}
$$

Thus we have (IV.6) if $\delta \epsilon<1$.

Proof of Theorem 5.3: (Outline only) Since the $l$-th row of $N^{\operatorname{adj}}\left(z_{i}\right)$ is $k_{l} v_{i}$ for some $k_{l} \in \mathbb{C}$ by the proof of Theorem 3.5 , we can show that $v_{i}^{*}\left(I-D\left(z_{i}\right) Y\left(z_{i}\right)\right)=0$.

On the other hand, if there exists $Y_{o} \in\left(H^{\infty}\right)^{p \times p}$ such that

$$
v_{i}^{*}\left(I-f\left(z_{i}\right) D\left(z_{i}\right) Y_{o}\left(z_{i}\right)\right)=0,
$$

then $X_{o}:=N^{-1}\left(I-f D Y_{o}\right)$ is in $\left(H^{\infty}\right)^{p \times p}$ and satisfies

$$
N X_{o}+f D Y_{o}=I \text {. }
$$

Hence it suffices to find $Y_{o} \in\left(H^{\infty}\right)^{p \times p}$ satisfying $Y_{o}\left(z_{i}\right)=$ $1 / f\left(z_{i}\right) \cdot Y\left(z_{i}\right)$ for every $i$. This is possible by Lagrange interpolation [4].

\section{REFERENCES}

[1] J. A. Ball, I. Gohberg, and L. Rodman. Interpolation of Rational Matrix Functions. Birkhäuser-Verlag, Basel, 1990.

[2] A. Cavallo, G. De Maria, C. Natale, and S. Pirozzi. Robust control of flexible structures with stable bandpass controllers. Automatica, 44:1251-1260, 2008.

[3] C. Cosner, G. Anwar, and M. Tomizuka. Plug in repetitive control for industrial robotic manipulators. In Proc. of IEEE International Conference on Robutics and Automation, 1990.

[4] P. Dorate. Analytic Feedback Design: An Interpolation Approach. Pacific Grove, CA: Brooks/Col, 2000.

[5] J. C. Doyle, B. A. Francis, and A. Tannenbaum. Feedback Control Theory. New York: Macmillan, 1992.

[6] C. Foiaş and A. E. Frazho. The Commutant Lifting Approach to Interpolation Problems. Birkhäuser-Verlag, Basel, 1990.

[7] C. Foiaş, H. Özbay, and A. Tannenbaum. Robust Control of Infinite Dimensional Systems: Frequency Domain Methods. Lecture Notes in Control and Information Sciences, No. 209, Springer-Verlag, London, 1996.
[8] B. A. Francis and W. M. Wonham. The internal model principle for linear multivariable regulators. Applied Mathematics \& Optimization, 2:170-194, 1975.

[9] C. Ganesh. Synthesis of optimal control systems with stable feedback. $\mathrm{PhD}$ thesis, Rice University, 1987.

[10] R. Griñó, R. Cardoner, R. Costa-Castelló, and E. Fossas. Digital repetitive control of a three-phase four-wire shunt active filter. IEEE Transactions on Industrial Electronics, 54:1495-1503, 2007.

[11] S. Gümüşsoy and H. Özbay. Remarks on strong stabilization and stable $\mathcal{H}^{\infty}$ controller design. IEEE Transactions on Automatic Control, 50:2083-2087, 2005.

[12] S. Gümüşsoy and H. Özbay. Stable $\mathcal{H}^{\infty}$ controller design for timedelay systems. International Journal of Control, 81:546-556, 2008.

[13] S. Gümüşsoy and H. Özbay. Sensitivity minimization by strongly stabilizing controllers for a class of unstable time-delay systems. IEEE Transactions on Automatic Control, 54:590-595, 2009.

[14] S. Hara, Y. Yamamoto, T. Omata, and M. Nakano. Repetitive control system: A new type servo system for periodic exogenous signals. IEEE Transactions on Automatic Control, 33:659-668, 1988.

[15] I. Houtzager, J.-W. van Wingeren, and M. Verhaegen. Rejection of periodic wind disturbances on a smart rotor test section using lifted repetitive control. To appear in IEEE Transactions on Control Systems Technology, 2012.

[16] H. Ito, H. Ohmori, and A. Sano. Design of stable controllers attaining low $\mathscr{H}^{\infty}$ weighted sensitivity. IEEE Transactions on Automatic Control, 38:485-488, 1993.

[17] H. Kimura. Directional interpolation approach to $H^{\infty}$-optimization and robust stabilization. IEEE Transactions on Automatic Control, 32:1085-1093, 1987.

[18] J.-H. Moon, M.-N. Lee, and M. J. Chung. Repetitive control for the track-following servo system of an optical disk drive. IEEE Transactions on Control Systems Technology, 6:663-670, 1998.

[19] Y. Ohta, H. Maeda, and S. Kodama. Unit interpolation in $H_{\infty}$ : Bounds of norm and degree of interpolants. Systems \& Control Letters, 17:251-256, 1991.

[20] H. Özbay. Stable $\mathcal{H}^{\infty}$ controller design for systems with time delays. In Perspectives in Mathematical System Theory, Control, and Signal Processing: A Festschrift in Honor of Yutaka Yamamoto on the Occasion of His 60th Birthday, pages 105-113. Springer-Verlag, 2010.

[21] J. R. Partington and P. M. Mäkilä. Rational approximation of distributed-delay controllers. International Journal of Control, 78:1295-1301, 2005.

[22] I. R. Petersen. Robust $H^{\infty}$ control of an uncertain system via a stable output feedback controller. IEEE Transactions on Automatic Control, 54:1418-1423, 2009.

[23] R. K. Prasanth. Two-sided tangential interpolation with real rational units in $\mathscr{H}^{\infty}$. Automatica, 34:861-874, 1998.

[24] A. Quadrat. On a general structure of the stabilizing controllers based on stable range. SIAM Journal on Control and Optimization, 42:22642285,2004

[25] J. Shi and W. S. Lee. Analytical feedback design via interpolation approach for the strong stabilization of a magnetic bearing system. In Proc. of Chinese Control and Decision Conference, 2009.

[26] M. C. Smith. On stabilization and the existence of coprime factorizations. IEEE Transactions on Automatic Control, 34:1005-1007, 1989.

[27] H. U. Ünal and A. İftar. Stable $\mathcal{H}^{\infty}$ flow controller design using approximation of FIR filters. Transactions of the Institute of Measurement and Control, 34:3-25, 2012.

[28] M. Wakaiki, Y. Yamamoto, and H. Özbay. Sensitivity reduction by strongly stabilizing controllers for MIMO distributed parameter systems. IEEE Transactions on Automatic Control, 57:2089-2094, 2012.

[29] G. Weiss, Q.-C. Zhong, T. C. Green, and J. Liang. $H^{\infty}$ repetitive control of DC-AC converters in microgrids. IEEE Transactions on Power Electronics, 19:219-230, 2004.

[30] Y. Yamamoto. Equivalence of internal and external stability for a class of distributed systems. Mathematics of Control, Signals, and Systems, 4:391-409, 1991.

[31] Y. Yamamoto and S. Hara. Relationships between internal and external stability for infinite-dimensional systems with applications to a servo problem. IEEE Transactions on Automatic Control, 33:1044-1052, 1988.

[32] M. Zeren and H. Özbay. On the strong stabilization and stable $H^{\infty}$ controller design problems for MIMO systems. Automatica, 36:16751684,2000 